\title{
Design of 45nm Switched Inverter Scheme (SIS) ADCs for Low Power and High Speed Applications
}

\author{
Arun Kumar Sunaniya, \\ ECE, MANIT, Bhopal, India
}

\author{
Kavita Khare \\ ECE, MANIT, Bhopal, India
}

\begin{abstract}
The Analog to Digital converters (ADC) play a very important role in today's world of electronic systems. The requirement of present applications demands high speed, low power dissipation, minimum area, low noise and application specific resolution. Out of the various types of ADCs available the flash ADC is most popular for its highest conversion rate and its wide applications. On the down side the flash ADC dissipates high power due to the presence of resistance ladder. The power dissipation further increases with increase in resolution. In this research two different approaches are presented which eliminates the resistor ladder completely and hence reduce the power demand drastically. The first approach is Switched Inverter Scheme (SIS) ADC; it is realized for 3 bits using 7 comparator circuits of varying size in CMOS $45 \mathrm{~nm}$ technology with Predictive Technology Model (PTM). The test result obtained indicates an offset error of $0.014 \mathrm{LSB}$. The full scale error is of $-0.112 \mathrm{LSB}$. The gain error is of $0.07 \mathrm{LSB}$, actual full scale range of $0.49 \mathrm{~V}$, worst case DNL \& INL each of $-0.3 \mathrm{~V}$. The power dissipation for the SIS ADC is $207.987 \mu$ watts; Power delay product (PDP) is $415.9 \mathrm{fWs}$, and the area overhead is $1.89 \mu \mathrm{m}^{2}$. The second approach is Sleep transistor SIS ADC. This approach shows $71 \%$ improvement in power dissipation. Whereas PDP is found to be $107.3 \mathrm{fWs}$ and area overhead is $1.94 \mu \mathrm{m} 2$ for Sleep transistor SIS ADC.
\end{abstract}

\section{General Terms}

ADC, Switched Inverter Scheme (SIS)

\section{Keywords}

CMOS $45 \mathrm{~nm}$, flash analog to digital converter, low power, resistor less, switched inverter scheme (SIS), sleep transistor

\section{INTRODUCTION}

Recent advances in the field of electronics require data converters for interfacing the signals of real world and then processing the obtained information in digital domain [10], [20]. In present scenario an ADC became an unavoidable component and it is also required to be fabricated on the same chip instead of a separate circuit for data conversion.

Since a few years lot of research is done on data converters to achieve maximum speed and minimum power consumption [8]. As technology scaling continues the demand of minimum power dissipation further increases in various modern battery powered applications [25].
The research is continuing in various types of data converters like flash, semi flash, sigma delta, pipeline, folding, and successive approximation register (SAR) ADCs to optimize their performance [1]. The insatiable demand of high performance data converters attracts researchers due to their wide usage in digital signal processing. In various applications data converters repress the performance severely if not designed optimally. Investigation of new design techniques for an $\mathrm{ADC}$ is in progress in order to reduce power dissipation, increase operating speed and decrease chip area [3].

High speed data converters are the key building blocks in many applications including high data rate serial links [2], [11]. Ultra wide band systems [4], magnetic and optical data storage devices [5], high speed instrumentation, wideband radar and optical communications [8], [12], 3G telecommunication systems, wireless wide area networks, broadband wireless communication network, radio astronomy, optical communication systems [6]. ADCs with high speed and resolution in the range of 6-8 bits are used in digital telecommunications, cryptography, high performance image sensing and processing, digital signal oscilloscopes and gigabit Ethernet. ADCs with low power dissipation are especially required for system on chip (SOC) applications, cellular phones, Digital TVs, \& spectrum monitoring systems [7].

Out of various ADC architectures available, the design of flash ADC becomes quite more important as it is used in other ADC architectures like two step ADC and multi bit sigma delta ADC. The block diagram of a flash ADC is as shown in Fig.1. [26], [38].

Due to increase in number of resistors and comparators required in designing of fully parallel flash ADCs having resolution 8 bits and above, the layout area \& power dissipation also increases. Therefore in recent years the research is carried with resistor less flash ADC architecture.

New methods developed to reduce the power consumption of flash ADCs are [5].

Due to increase in number of resistors and comparators required in designing of fully parallel flash ADCs having resolution 8 bits and above, the layout area \& power dissipation also increases. Therefore in recent years the research is carried with resistor less flash ADC architecture.

New methods developed to reduce the power consumption of flash ADCs are [5]. 


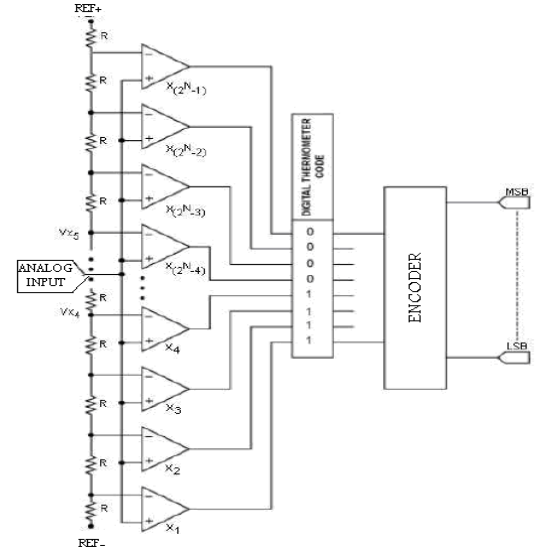

Fig.1. Block Diagram of a Flash ADC

- Use of interpolation and voltage to current converters that operate as preamplifier stage of latches.

- Extension in the input range.

- Use of bisection method to let only half of the ADCs working in every clock cycle.

The aim of this work is to design the ADCs required for battery operated devices. The ADCs are designed and simulated with two different power saving schemes. The main improvements for various schemes are envisaged in power dissipation, differential and integral non linearity. The specifications of the design prototype are selected as 3 bit, for $500 \mathrm{MHz}$ signal, and $0.5 \mathrm{~V}$ full scale range, with a supply voltage of $1 \mathrm{~V}$. The simulation is carried out in $45 \mathrm{~nm}$ PTM [39] on LT Spice IV platform.

In section II two different types of flash comparator architectures are discussed along with the mathematical relations for sizing the transistors of comparator. The design issues of complete design along with component blocks like gain booster, encoder are given in section III. In section IV results and discussions are presented. Section V concludes the work.

\section{DIFFERENT TYPES OF FLASH COMPARATORS}

\section{1 switched inverter scheme (SIS) comparator}

The SIS comparator has very simple architecture as shown in Fig.2. It is quite different than the conventional operational amplifier based differential input voltage (DIV) comparator [9], [29], [34]. The key difference between the DIV comparator and the switched inverter scheme (SIS) comparator scheme lies in the way the reference voltage is generated for each level. In DIV comparator conventional method of using resistor ladder is utilized for externally generating the reference voltage. Where as in the SIS comparator scheme all $2 \mathrm{n}-1$ reference voltage for a $\mathrm{n}$ bit ADC are set internally by adjusting the threshold voltage of each voltage comparator separately by sizing the transistor properly. All DIV comparators are identical and duplicated for $2 \mathrm{n}-1$ times but each SIS comparator is altogether different from others and obtained by varying the $B$ ratio of the inverters.

The SIS comparator design consists of two pairs of inverters connected back to back. Each of the inverter is sized separately to get a unique switching voltage [13], [17].
The cascaded inverters then work as voltage comparator. The full scale voltage range (VFSR) is equally divided by $2 \mathrm{n}-1$ SIS comparators, to obtain the switching point voltage for each comparator separately.

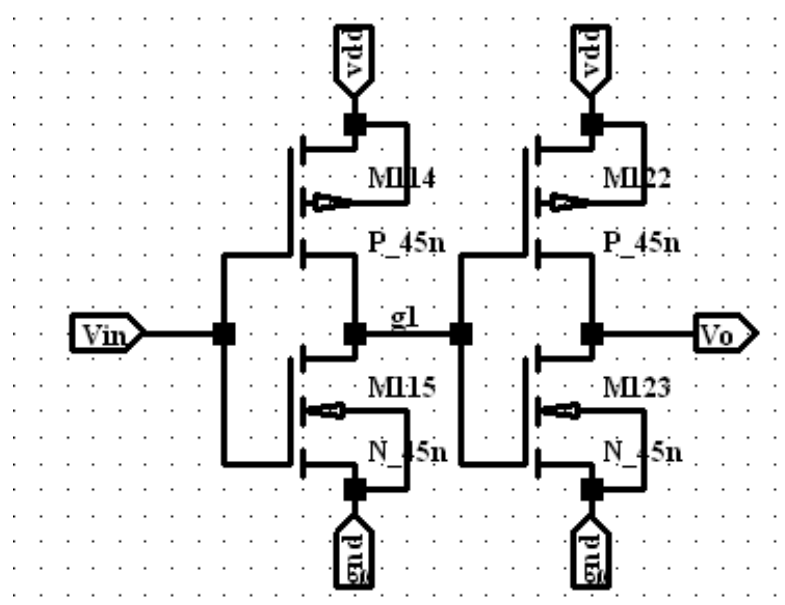

Fig.2. SIS Comparator

Achieving optimal sizing for each transistor in SIS comparator scheme is very much time consuming. The analytical expression for $(\mathrm{Wp} / \mathrm{Wn})$ of each comparator is determined by equating the pull up and pull down saturation currents as equation (1) [14], [15], [16], [18]. $K_{p} V_{D S A T p}\left(V_{m}-V_{D D}-V_{t p}-\frac{V_{D S A T p}}{2}\right)$

(1) The expression for switching voltage $\mathrm{Vm}$ is thus obtained for a short channel CMOS inverter in $45 \mathrm{~nm}$ as equation (2) [19], [21], [22], [30].

$$
\mathrm{V}_{\mathrm{m}}=\frac{\mathrm{V}_{\mathrm{DD}}-\left|\mathrm{V}_{\mathrm{tp}}\right|+\mathrm{V}_{\mathrm{tn}} \sqrt{1 / \mathrm{r}}}{1+\sqrt{1 / \mathrm{r}}}
$$

Where,

$$
\begin{aligned}
& r=\frac{K_{p} V_{\text {DSATp }}}{K_{n} V_{\text {DSATn }}}=\frac{v_{\text {satp }} \cdot W_{p}}{v_{\text {satn }} \cdot W_{n}} \\
& r=\frac{\mu_{p}\left(W_{p} / L_{p}\right)}{\mu_{n}\left(W_{n} / L_{n}\right)} \\
& K_{p}=K_{p}\left(\frac{W}{L}\right)=\beta_{p} \\
& K_{p}=\mu_{p} \cdot C_{\text {oxp }} \\
& K_{n}=K_{n}\left(\frac{W}{L}\right)=\beta_{n} \\
& K_{n}=\mu_{n} \cdot C_{o x n}
\end{aligned}
$$

The above equations are realized on MATLAB 7.3.0 R2006b for switching voltage calculations. The program is given in Fig.3. 
$>$ un $=0.032$

up $=0.0095$;

$\mathrm{vm}=0.3: 0.071: 0.801$

$\operatorname{vdd}=1$;

$\operatorname{vtn}=0.22$;

$\mathrm{vtp}=0.22$;

$\mathrm{b}=\operatorname{sprintf}\left(' \% .4 \mathrm{f}^{\prime}, \mathrm{W}\right)$;

$\mathrm{W}=(\mathrm{un} /(\mathrm{up}))^{*}((\mathrm{vm}-\mathrm{vtn}) . /(\mathrm{vdd}-\mathrm{vtp}-\mathrm{vm})) .^{\wedge} 2 ;$

\section{Fig.3. MATLAB code for switching voltage calculation}

The main advantage of the SIS comparators is that it does not use any resistor ladder and so it is suitable for low voltage, low power and high speed applications such as cellular mobile phones and touch screen display devices. On the other hand the process parameter variations may cause offset and gain errors to vary considerably when the design is used for higher resolution ADCs. Also noise can be a big challenge for the single ended SIS comparator scheme.

\subsection{SIS comparator with sleep transistor}

The circuit of SIS comparator is modified by addition of high threshold PMOS and NMOS near the supply rails as shown in Fig.4. The addition of header \& footer reduces the static power dissipation to a great extent due to increased resistance of the high threshold PMOS and NMOS transistors [27], [31], [36]. During the period of no activity the section not in use remains completely off. Whereas, the components are invoked again when any activity is detected.

A Local sleep transistor network is used here as opposed to global level network, because every comparator is differently sized and hence the current through each comparator section is also different. The sizing of a sleep transistor is calculated using equation (9).

$$
\left(\frac{\mathrm{W}}{\mathrm{L}}\right)_{\text {Sleep }}=\frac{\mathrm{I}_{\text {Sleep }}}{0.0281 \mu_{\mathrm{n}} \mathrm{C}_{\mathrm{ox}}\left(\mathrm{V}_{\mathrm{DD}}-\mathrm{V}_{\mathrm{tL}}\right)\left(\mathrm{V}_{\mathrm{DD}}-\mathrm{V}_{\mathrm{tH}}\right)}
$$

Where, Isleep is calculated by simulating the circuit without sleep transistor network and finding maximum current that flows through ground or VDD.

$$
\begin{aligned}
& \mathrm{C}_{\mathrm{ox}}^{\prime}=\frac{\varepsilon_{0} \varepsilon_{\mathrm{r}}}{\mathrm{t}_{\mathrm{ox}}} \\
& \mathrm{C}_{\mathrm{ox}}^{\prime}=\frac{\varepsilon_{\mathrm{ox}}}{\mathrm{t}_{\mathrm{ox}}} \\
& \mathrm{C}_{\mathrm{ox}}^{\prime}=\frac{\varepsilon_{0} \varepsilon_{\mathrm{ox}}}{\mathrm{t}_{\mathrm{ox}}} \\
& \mathrm{C}_{\mathrm{ox}}=\mathrm{C}_{\mathrm{ox}}^{\prime} \cdot \mathrm{A} \\
& \mathrm{A}=\mathrm{W} \cdot \mathrm{L}
\end{aligned}
$$

All the symbols used in equation (1) to (14) have

\begin{tabular}{|c|c|c|c|c|c|c|c|c|c|c|c|c|c|}
\hline \multicolumn{7}{|c|}{$\begin{array}{c}\text { Input Thermometer } \\
\text { code }\end{array}$} & \multicolumn{7}{|c|}{ Output "01" code } \\
\hline $\mathbf{T}$ & $\mathbf{T}$ & $\mathbf{T}$ & $\mathbf{T}$ & $\mathbf{T}$ & $\mathbf{T}$ & $\mathbf{T}$ & $\mathbf{G}$ & G & $\mathbf{G}$ & G & G & $\mathbf{G}$ & $\mathbf{G}$ \\
\hline 7 & 6 & 5 & 4 & 3 & 2 & 1 & 7 & 6 & 5 & 4 & 3 & 2 & 1 \\
\hline 0 & 0 & 0 & 0 & 0 & 0 & 0 & 0 & 0 & 0 & 0 & 0 & 0 & 1 \\
\hline 0 & 0 & 0 & 0 & 0 & 0 & 1 & 0 & 0 & 0 & 0 & 0 & 1 & 0 \\
\hline 0 & 0 & 0 & 0 & 0 & 1 & 1 & 0 & 0 & 0 & 0 & 1 & 0 & 0 \\
\hline 0 & 0 & 0 & 0 & 1 & 1 & 1 & 0 & 0 & 0 & 1 & 0 & 0 & 0 \\
\hline 0 & 0 & 0 & 1 & 1 & 1 & 1 & 0 & 0 & 1 & 0 & 0 & 0 & 0 \\
\hline 0 & 0 & 1 & 1 & 1 & 1 & 1 & 0 & 1 & 0 & 0 & 0 & 0 & 0 \\
\hline 0 & 1 & 1 & 1 & 1 & 1 & 1 & 1 & 0 & 0 & 0 & 0 & 0 & 0 \\
\hline 1 & 1 & 1 & 1 & 1 & 1 & 1 & 0 & 0 & 0 & 0 & 0 & 0 & 0 \\
\hline
\end{tabular}
usual meaning and are also universally known.

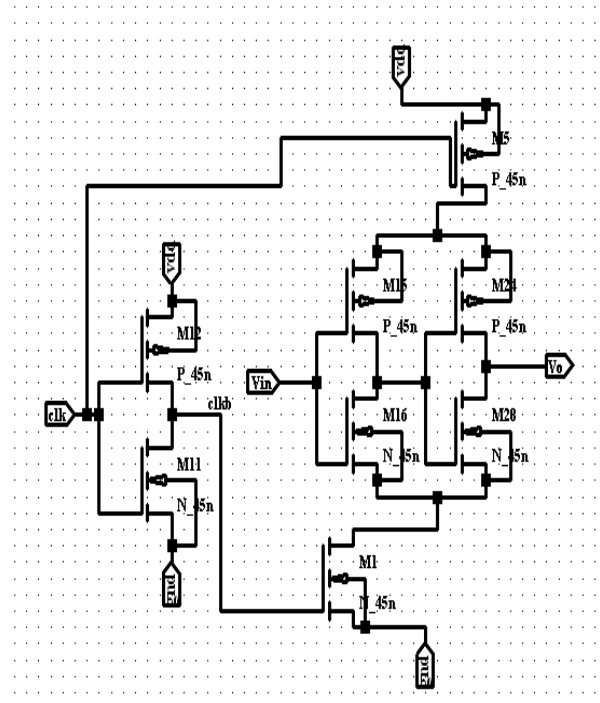

Fig.4. SIS Comparator with Sleep Transistor

Table I

Truth table for one out of $\mathbf{n}$ code

\section{DESIGN OF ADCs}

For the complete realization of any flash ADC the blocks required other than comparator are gain booster and thermometer to binary encoder. The design of these blocks is given here.

\subsection{Gain Booster}

The gain booster is used to increase voltage gain of the output of a comparator so that it provides a full digital output voltage swing, without which the output of the comparator circuit is unable to drive the next stage, it also makes thresholds sharper for comparator outputs and provide full digital output voltage swing. The gain booster block is designed for lowest switching voltage.

Each gain booster consists of two identical cascaded inverters with the same circuit as that of comparator, but the transistors are sized for minimum aspect ratio for each gain booster [23]. 


\subsection{Thermometer code converter}

The encoder converts the thermometer code to binary code in two steps. In the first step the thermometer code is converted into one out of $n$ code by using the truth table as shown in Table 1 . The one out of $\mathrm{n}$ codes is then converted to binary code $\mathrm{d} 2, \mathrm{~d} 1$, d0 by Read only memory (ROM) encoder, as shown in Fig.5. [35]. The ROM encoder is a common and straight forward approach to encode the one out of $n$ code to binary bit. The appropriate row $\mathrm{m}$ in the ROM is selected by using a row decoder that has the output of comparator $m$ and the inverse of comparator $m+1$ as inputs. The output $m$ of the row decoder, connected to memory row $\mathrm{m}$, is high if the output of comparator $\mathrm{m}$ is high and the output of comparator $\mathrm{m}+1$ is low. The row decoder can be realized by, a number of 2-input NAND gates, where one input to each NAND gate is inverted. The main advantage of the ROM decoder approach is its regular structure that is easy to design [34].

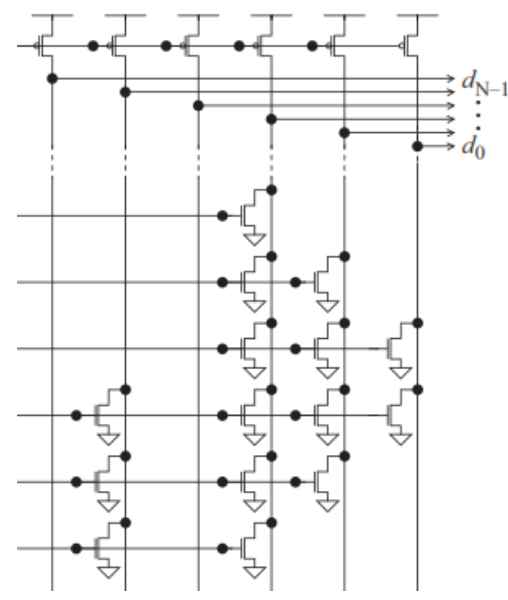

Fig.5. ROM Encoder

\section{RESULTS AND DISCUSSION}

The proposed technique is applied in the design of 3 bit ADC with chosen specifications as given in Table II and the implementation is as shown in Fig.6 for SIS ADC \& Fig. 8 for sleep transistor ADC [23], [24].

The design consists of comparator chain, gain booster circuit and a thermometer to binary encoder block and finally a buffer circuitry to reduce the loading effects.

Conventional voltage comparators use a resistor ladder circuit to generate 2n-1reference voltages (Vref) for comparators, ranging from Vref $(\max )$ to $\operatorname{Vref}(\min )$. Generating equally spaced reference voltages determines the quantization performance of ADCs [9], [21]. The SIS comparator eliminates the use of the resistor ladder circuit by generating the reference voltages internally. The SIS comparator uses two cascaded inverters to generate the range of internal reference voltages. At the first inverter, the analog input quantization is determined by adjusting the ratio of the PMOS and NMOS transistors. The second inverter is used to increase the voltage gain and prevent unbalanced propagation delays of all comparators.
The results for SIS Comparator are shown in Fig. 7 and for the modified Sleep transistor version in Fig.9.

The total power consumption here is determined by summing up the power dissipation of each circuit separately. The various blocks used to realize the design are comparator section, gain booster, XOR gate, ROM Encoder and buffers. The comparison results are shown in Table III for both types of ADCs [17], [31], [41], [42].

TABLE II

ADC SPECIFICATION

\begin{tabular}{|c|c|c|}
\hline S No. & Specification & Value \\
\hline 1 & Resolution & 3 Bit \\
\hline 2 & CMOS tech. & $45 \mathrm{~nm}$ \\
\hline 3 & Model File & PTM \\
\hline 4 & $\mathrm{~L}_{\mathrm{P}}=\mathrm{L}_{\mathrm{N}}=\mathrm{L}_{\min }$ & $45 \mathrm{~nm}$ \\
\hline 5 & $t_{o x}$ & $14 \AA$ \\
\hline 6 & $\mathrm{C}_{\mathrm{ox}}$ & $25 \mathrm{fF} / \mu \mathrm{m}^{2}$ \\
\hline 7 & Max. Speed & 500 MSPS \\
\hline 8 & $\mathrm{~V}_{\mathrm{FSR}}$ & $0.3 \mathrm{~V}-0.8 \mathrm{~V}$ \\
\hline 9 & $V_{\text {LSB }}$ & $0.0714 \mathrm{~V}$ \\
\hline 10 & $\left(\mathrm{~W}_{\mathrm{p}} / \mathrm{W}_{\mathrm{n}}\right)_{\text {Min }}$ & 0.55 \\
\hline 11 & $\left(\mathrm{~W}_{\mathrm{p}} / \mathrm{W}_{\mathrm{n}}\right)_{\mathrm{Max}}$ & 266 \\
\hline 12 & $\mathrm{~V}_{\mathrm{DD}}$ & $1 \mathrm{~V}$ \\
\hline 13 & $\mathrm{~V}_{\mathrm{TP}}$ & $-0.22 \mathrm{~V}$ \\
\hline 14 & $\mathrm{~V}_{\mathrm{TN}}$ & $0.22 \mathrm{~V}$ \\
\hline 15 & $\mathrm{~V}_{\mathrm{m} \min }$ & $0.371 \mathrm{~V}$ \\
\hline 16 & $V_{m \max }$ & $0.8 \mathrm{~V}$ \\
\hline 17 & $\mu_{n}$ & $320 \mathrm{~cm}^{2} / \mathrm{v}-\mathrm{sec}$ \\
\hline 18 & $\mu_{p}$ & $95 \mathrm{~cm}^{2} / \mathrm{v}$-sec \\
\hline 19 & $\begin{array}{c}\mathrm{C}_{\mathrm{L}}(\text { Load } \\
\text { Capacitance })\end{array}$ & $0.01 \mathrm{fF}$ \\
\hline
\end{tabular}




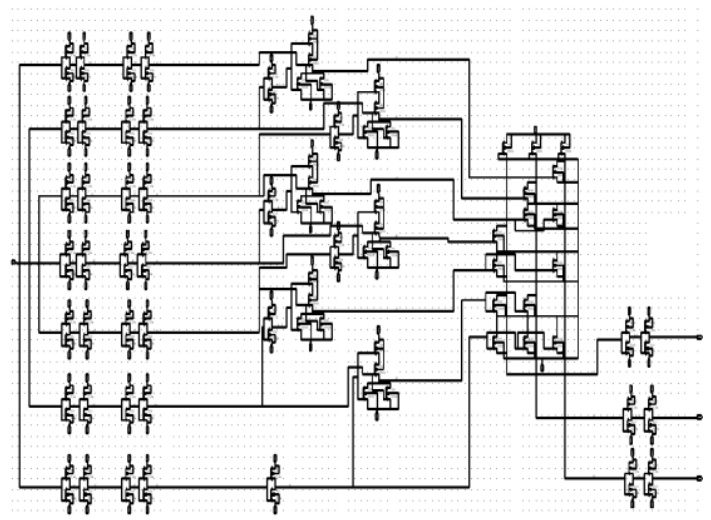

Fig.6. SIS ADC with ROM Encoder

The power dissipation is calculated and is compared for both the designed ADCs. The finding for saving in power dissipation is shown in Table IV.

The various dynamic parameters for all ADCs are also calculated using the equations (15) - (22) [30], [40] and are tabulated in Table V.

Offset error $=$ real transition - ideal transition (15)

Offset in LSB = offset error/ One LSB

(16)

\subsection{Design of SIS ADC}

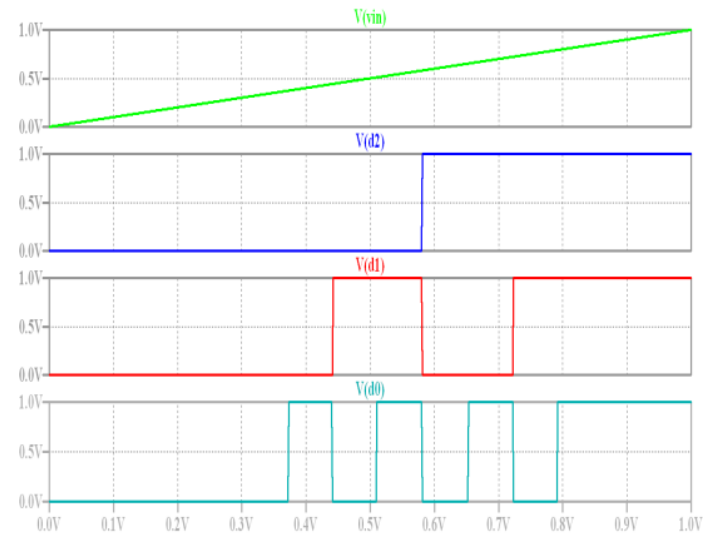

Fig.7. Simulation Results of SIS ADC

\subsection{Design of Sleep Transistor SIS ADC}

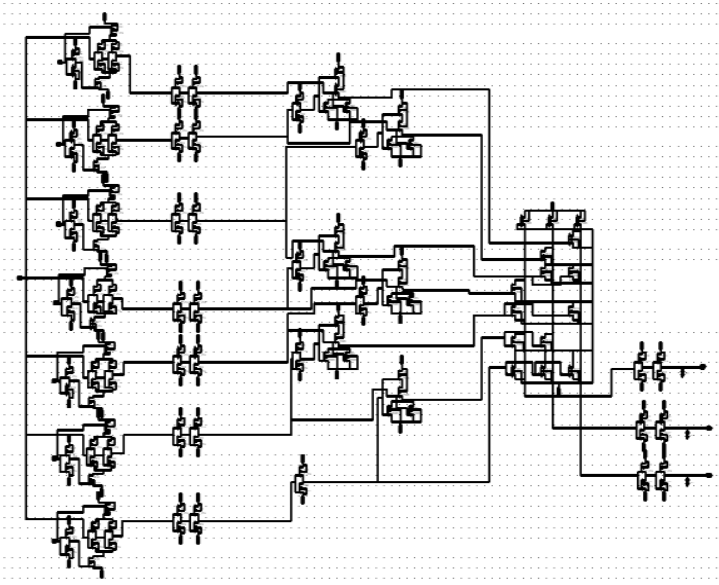

Fig.8. Sleep Transistor SIS ADC with ROM Encoder

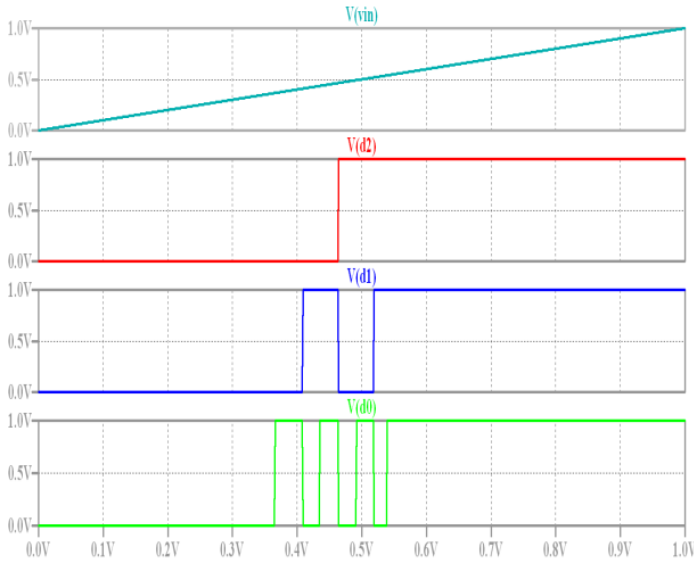

Fig.9. Simulation Results of Sleep Transistor SIS ADC

Full Scale Error $=$ real last transition - ideal last

$$
\text { transition }
$$

Gain error $=$ Average code width $($ LSB size $)$

$=\underline{\text { Real last transition }- \text { first transition }}$

(18) $\quad 2\left(2^{\mathrm{N}-1}-1\right)$

$$
\mathrm{DNL}=\mathrm{DNL}[\mathrm{k}]=\text { Width }[\mathrm{k}]-1
$$

TABLE III

COMPARISON OF COMPONENT POWER DISSIPATION IN $\boldsymbol{\mu W}$

\begin{tabular}{|c|l|c|c|}
\hline S.No. & Blocks & $\begin{array}{c}\text { SIS ADC } \\
{[17],[31],} \\
{[41],[42]}\end{array}$ & $\begin{array}{c}\text { Sleep } \\
\text { SIS } \\
\text { ADC }\end{array}$ \\
\hline 1 & Comparator & 167.087 & 37.88 \\
\hline 2 & $\begin{array}{l}\text { Gain } \\
\text { Booster }\end{array}$ & 35.18 & 18.48 \\
\hline 3 & $\begin{array}{l}\text { One out of } \\
\text { n coder }\end{array}$ & 3.1357 & 1.8 \\
\hline 4 & $\begin{array}{l}\text { ROM } \\
\text { encoder }\end{array}$ & 2.153 & 1.09 \\
\hline 5 & Buffers & 0.437 & 0.31 \\
\hline 6 & $\begin{array}{l}\text { Total } \\
\text { Power }\end{array}$ & 207.987 & 59.64 \\
\hline 7 & Delay & $2 \mathrm{~ns}$ & $1.8 \mathrm{~ns}$ \\
\hline 8 & $\begin{array}{l}\text { Power } \\
\text { Delay } \\
\text { Product }\end{array}$ & $415.9 \mathrm{fWs}^{107.3}$ \\
\hline 9 & Area & $1.89 \mu \mathrm{m}^{2}$ & $\begin{array}{l}1.94 \\
\mathrm{~m}^{2}\end{array}$ \\
\hline
\end{tabular}


TABLE IV

BLOCK WISE PERCENTAGE SAVING IN POWER DISSIPATION AS COMPARED TO SIS ADC

\begin{tabular}{|c|l|l|}
\hline S.No. & Blocks & $\begin{array}{c}\text { Sleep SIS } \\
\text { ADC }\end{array}$ \\
\hline 1 & Comparator & $77.3 \%$ \\
\hline 2 & $\begin{array}{l}\text { Gain } \\
\text { Booster }\end{array}$ & $47.47 \%$ \\
\hline 3 & $\begin{array}{l}\text { One out of } \\
\text { n coder }\end{array}$ & $42.6 \%$ \\
\hline 4 & $\begin{array}{l}\text { ROM } \\
\text { encoder }\end{array}$ & $49.37 \%$ \\
\hline 5 & \begin{tabular}{l} 
Buffers \\
\hline 6
\end{tabular} & $\begin{array}{l}\text { Total } \\
\text { Power }\end{array}$ \\
\hline
\end{tabular}

Where,

Width $[\mathrm{k}]=$ Transition $[\mathrm{k}+1]-$ Transition $[\mathrm{k}](20)$

$\mathrm{INL}=$

$\operatorname{INL}(\mathrm{m})=\underline{\text { Transition }[\mathrm{m}]-\text { Transition[ideal] }}(21)$
Step Width [Ideal]

Table V

DYNAMIC PARAMETERS FOR ADCS IN LSB

\begin{tabular}{|c|c|c|}
\hline Parameter & $\begin{array}{c}\text { SIS } \\
\text { ADC }\end{array}$ & $\begin{array}{c}\text { Sleep } \\
\text { Transistor } \\
\text { SIS ADC }\end{array}$ \\
\hline Offset Error & 0.014 & -0.084 \\
\hline $\begin{array}{c}\text { Full Scale } \\
\text { Error }\end{array}$ & -0.112 & -0.36 \\
\hline Gain Error & 0.07 & 0.021 \\
\hline DNL & -0.3 & -0.6 \\
\hline INL & -0.3 & -0.4 \\
\hline
\end{tabular}

$$
\mathrm{INL}=\sum_{i=1}^{m} D N L[i]
$$

DNL is Differential non linearity error and INL is Integral non linearity error. The quantization error in each case is less than $0.5 \mathrm{LSB}$.

Comparison of this work with existing ADCs is shown in Table VI.

Table VI

COMPARISON WITH EXISTING ADCs

\begin{tabular}{|c|c|c|c|c|c|c|c|c|c|c|c|}
\hline S.No & $\begin{array}{c}\text { Publicat } \\
\text { ion }\end{array}$ & Year & Type & $\begin{array}{c}\text { No. } \\
\text { of } \\
\text { Bits }\end{array}$ & $\begin{array}{c}\text { Sampling } \\
\text { Frequency } \\
\text { (SPS) }\end{array}$ & $\begin{array}{c}\text { Suppl } \\
\text { y } \\
\text { Voltag } \\
\text { e }\end{array}$ & $\begin{array}{c}\text { Technolo } \\
\text { gy }\end{array}$ & $\begin{array}{l}\text { DNL } \\
\text { (LSB) }\end{array}$ & $\begin{array}{c}\text { INL } \\
\text { (LSB) }\end{array}$ & SNR & $\begin{array}{c}\text { Power } \\
\text { Dissipati } \\
\text { on }\end{array}$ \\
\hline 1. & $\begin{array}{c}\text { This } \\
\text { Work }\end{array}$ & - & $\begin{array}{l}\text { Flash } \\
\text { (SIS } \\
\text { ADC) }\end{array}$ & 3 & $500 \mathrm{M}$ & $1 \mathrm{~V}$ & $45 \mathrm{~nm}$ & $+/-0.3$ & $+/-0.3$ & - & $\begin{array}{c}207.98 \\
\mu \mathrm{W}\end{array}$ \\
\hline 2. & $\begin{array}{c}\text { This } \\
\text { Work }\end{array}$ & - & $\begin{array}{c}\text { Flash } \\
\text { (Sleep } \\
\text { Transi } \\
\text { stor } \\
\text { SIS } \\
\text { ADC) }\end{array}$ & 3 & $500 \mathrm{M}$ & $1 \mathrm{~V}$ & $45 \mathrm{~nm}$ & $+/-0.6$ & $+/-0.4$ & - & $\begin{array}{c}59.64 \\
\mu \mathrm{W}\end{array}$ \\
\hline 3. & [12] & 2011 & SAR & 6 & $50 \mathrm{M}$ & $1 \mathrm{~V}$ & $90 \mathrm{~nm}$ & $<0.64 \mathrm{LSB}$ & $<0.54 \mathrm{LSB}$ & $32 \mathrm{~dB}$ & $240 \mathrm{uW}$ \\
\hline 4. & [13] & 2011 & Flash & 4 & $200 \mathrm{M}$ & $3.3 \mathrm{v}$ & $0.35 \mathrm{um}$ & $\begin{array}{c}0.2 \mathrm{LSB} /- \\
0.9 \mathrm{LSB}\end{array}$ & 0.2 & $20 \mathrm{~dB}$ & $12.4 \mathrm{~mW}$ \\
\hline 5. & [26] & 2010 & Flash & 4 & $1.9 \mathrm{G}$ & $1.8 \mathrm{~V}$ & $0.5 \mathrm{um}$ & $\begin{array}{c}+0.16 /- \\
0.16 \mathrm{LSB}\end{array}$ & $\begin{array}{c}+0.16 /- \\
0.104\end{array}$ & - & $\begin{array}{c}0.28573 \\
\mathrm{~mW}\end{array}$ \\
\hline 6. & [28] & 2010 & SAR & 9 & $80 \mathrm{M}$ & $1 \mathrm{~V}$ & $65 \mathrm{~nm}$ & 0.37 & 0.40 & $\begin{array}{c}50.71 \\
\mathrm{~dB}\end{array}$ & $3.4 \mathrm{~mW}$ \\
\hline 7. & [31] & 2009 & Flash & 3 & - & $3.3 \mathrm{~V}$ & $130 \mathrm{~nm}$ & - & - & - & - \\
\hline 8. & [33] & 2008 & $\begin{array}{c}\text { Fold } \\
\text { ing }\end{array}$ & 9 & $1 \mathrm{G}$ & $3.3 \mathrm{~V}$ & $0.35 \mathrm{um}$ & - & - & - & $375 \mathrm{~mW}$ \\
\hline 9. & [32] & 2008 & Flash & $3-6$ & $200 \mathrm{M}$ & $3.3 \mathrm{v}$ & 0.35 um & $0.519 \mathrm{LSB}$ & $0.423 \mathrm{LSB}$ & - & - \\
\hline 10. & [35] & 2007 & $\begin{array}{l}\text { Pipeli } \\
\text { ned } \\
\text { Flash }\end{array}$ & 4 & $2.5 \mathrm{G}$ & $1.2 \mathrm{~V}$ & $130 \mathrm{~nm}$ & 0.26 & 0.296 & - & $\begin{array}{c}23.778 \\
\mathrm{~mW}\end{array}$ \\
\hline 11. & [37] & 2006 & Flash & 6 & $500 \mathrm{M}$ & $3.3 \mathrm{~V}$ & 0.35 um & +0.2 & -0.2 & $\begin{array}{c}37.2 d \\
B\end{array}$ & - \\
\hline
\end{tabular}




\section{CONCLUSION}

Low power architecture for a 3-bit CMOS SIS based flash ADC is presented using PTM $45 \mathrm{~nm}$. The modified versions of SIS ADC can further achieve very low power dissipation; this proposed method can reduce power dissipation upto $71 \%$. It uses smaller silicon area of $1.94 \mu \mathrm{m} 2$. The DNL of the proposed $\mathrm{ADC}$ is within -0.3LSB and INL is also within $0.3 \mathrm{LSB}$. The proposed ADC chip so designed can be used for capacitive pressure sensor, video systems as well as it can be used in a low power two-step ADC, pipelined ADC and multi-bit sigma delta ADC. A disadvantage is that for higher no of bits the increased complexity of the circuit consumes more chip area and will likely consume more power.

\section{REFERENCES}

[1] Amol Inamdar, Anubhav Sahu, Jie Ren, Aniruddha Dayalu, and Deepnarayan Gupta, "Flash ADC Comparators and Techniques for their Evaluation", IEEE Transactions on Applied Superconductivity, Vol.23, no.3, ISSN No.1051-8223,pp. 1-8, Jan 2013.

[2] Xiangliang Jin, Zhibi Liu, and Jun Yang, "New Flash ADC Scheme With Maximal 13 Bit Variable Resolution and Reduced Clipped Noise for High-Performance Imaging Sensor", IEEE Sensors Journal, Vol. 13, no.1, pp. 167-171, Jan. 2013.

[3] Young-Kyun Cho, Jae-Ho Jung, and Kwang Chun Lee, "A 9-bit 100-MS/s Flash-SAR ADC without Track-andHold Circuits", International Symposium on Wireless Communication Systems (ISWCS),ISBN No. 978-14673-0761-1, pp.880-884, Aug 2012.

[4] Joyjit Mukhopadhyay and Soumya Pandit, " Modeling and Design of a Nano Scale CMOS Inverter for Symmetric Switching Characteristics", Hindawi Publishing Corporation VLSI Design, Vol.2012, pp.113,2012 .

[5] Chakir Mostafa,Hassan Qjidaa, "1 GS/s, Low Power FlashAnalog to Digital Converter in $90 \mathrm{~nm}$ CMOSTechnology", IEEE conference on Multimedia Computing and Systems (ICMCS), 2012 International ,pp.1097 - 1100,May2012.

[6] Yun-Shiang Shu, "A 6b 3GS/s 11mW Fully Dynamic Flash ADC in 40nm CMOS with Reduced Number of Comparators", IEEE Symposium on VLSI Circuits Digest of Technical Papers ,pp. 26-27, June2012.

[7] Ch. Vassou, L. Mountrichas, S. Siskos, "A NMOS Bulk Voltage Trimming Offset Calibration Technique for a 6bit 5GS/s Flash ADC", IEEE International Conference on Instrumentation and Measurement Technology (I2MTC),pp.5-8,May 2012.

[8] Soon-Kyun Shin, Jacques C. Rudell, Denis C. Daly, Carlos E. Muñoz, Dong-Young Chang, Kush Gulati, Hae-Seung Lee, and Matthew Z. Straayer, "A 12b 200MS/s Frequency Scalable Zero-Crossing Based Pipelined ADC in 55nm CMOS" ,IEEE Custom Integrated Circuits Conference (CICC), pp.1-4,2012.

[9] Yuji Gendai, and Akira Matsuzawa, "A Specific Distortion Pattern of Flash ADCs Identified by Discriminating Time-Domain Analysis", IEEE transactions on instrumentation and measurement, vol. 61, no. 2, pp.316-325, Feb. 2012.
[10] Amir Zjajo Jose, Pineda de Gyvez "Low-Power High Resolution Analog to Digital Converters, Design Test and Calibration" ISBN 978-90-481-9724-8, First edition, Springer, 2011

[11] Dharmendra Mani Varma "Reduced Comparator Low power Flash ADC using $35 \mathrm{~nm}$ CMOS", IEEE Conference on Electronics Computer Technology (ICECT) ,pp. 385- 388, April 2011.

[12] Pierluigi Nuzzo, Claudio Nani, Costantino Armiento, Alberto Sangiovanni Vincentelli, Jan Craninckx, Geert Van der Plas, "A 6-Bit 50-MS/s Threshold Configuring SAR ADC in 90-nm Digital CMOS",IEEE Trans. On Circuits And Systems-I,Vol.58, no. 12, December 2011.

[13] Sudakar S. Chauhan, S. Manabala, S.C. Bose and R. Chandel, "A New Approach To Design Low Power CMOS Flash A/D Converter", International Journal of VLSI design \& Communication Systems(VLSICS),Vol.2,no.2,pp.10-108,June 2011.

[14] A. Ávila, D. Espejo, "A SPICE-compatible Model for Intel's 45nm High K MOSFET", The World Congress on Engineering and Computer Science (WCECS) Proceedings, ISBN no.978-988-19251-7-6, Vol.2, pp.762-765, October 2011.

[15] G. Torfs, Z. Li, J. Bauwelinck, X. Yin, G. Van der Plas and J.Vandewege, "Low-power 4-bit flash analogue to digital converter for ranging applications", IEEE Electronics Letters, Vol. 47 no. 1, Jan. 2011.

[16] T. Esther Rani, Dr. Rameshwar rao, "Area And Power Optimized Multipliers With Minimum Leakage", IEEE International Conference on Electronic Computer Technology - ICECT , ISBN no.978-1-4244-8679,pp.284 -287 , Nov.2011.

[17] Oktay Aytar and Ali Tangel, "Employing threshold inverter quantization (TIQ) technique in designing 9-Bit folding and interpolation CMOS analog-to-digital converters (ADC)", Scientific Research and Essays,Vol.6,no.2, ISSN no. 1992-2248, pp. 351362,January 2011.

[18] Hiroshi Fuketa, Satoshilida, Tadashi Yasufuku, Makoto Takamiya, Masahiro Nomura, Hirofumi Shinohara, Takayasu Sakurai, "A Closed-form Expression for Estimating Minimum Operating Voltage (VDDmin) of CMOS Logic Gates", IEEE Conference on Design Automation (DAC),ISBN no. 978-1-4503-0636-2, pp.984-989, June 2011.

[19] Baozeng Guo, Tao Ma, Yubo Zhang "Design of A Novel Domino XNOR Gate for 32-nm node CMOS Technology", IEEE International conference on Electric Information and Control Engineering (ICEICE), ISBN no. 978-1-4244-8036-4, pp. 289-292 April 2011

[20] Tomoyuki Yamase, Hiroaki Uchida, and Hidemi Noguchi ,"A 22-mW 7b 1.3-GS/s Pipeline ADC with 1bit/stage Folding Converter Architecture", Symposium on VLSI Circuits Digest of Technical Papers, ISBN no. 978-4-86348-1657, pp.124-125,2011.

[21] Jaeyoon Kim, Sandip Tiwari, "Inexact Computing for Ultra Low Power Nanometer Digital Circuit Design", IEEE/ACM International Symposium on Nanoscale Architectures, 978-1-4577-0995-1, pp.24-31, Jan 2011. 
[22] Tsung-Ching Huang, Kenjiro Fukuda, Chun-Ming Lo, Yung-Hui Yeh, Tsuyoshi Sekitani, Takao Someya and Kwang-Ting Cheng, "Pseudo-CMOS: A Design Style for Low-Cost and Robust Flexible Electronics" IEEE Transactions on Electron Devices, Vol. 58, no.1, pp. 141150, Jan. 2011.

[23] Arun Kumar Sunaniya, Kavita Khare, "A Design Comparison of Low Power $50 \mathrm{~nm}$ Technology Based Inverter with Sleep Transistor and MTCMOS Scheme", International Journal of Engineering Science and Technology (IJEST), Vol. 3 No. 10, ISSN no. 09755462,pp. 7744-7753, October 2011.

[24] Arun Kumar Sunaniya, Kavita Khare, “A Low power 50 $\mathrm{nm}$ Technology Based CMOS Inverter with Sleep Transistor Scheme" International Journal of Computer Science Engineering \& Technology (IJCSET) Vol. 1 No. 9, ISSN no. 2231-0711,pp. 560-562, October 2011.

[25] The "International Technology Road map of semiconductor" ITRS, pp 61-66, 2010.

[26] Meghana Kulkarni, V. Sridhar, G.H. Kulkarni,"4-Bit Flash Analog to Digital Converter Design using CMOSLTE Comparator", IEEE Asia Pacific Conference on Circuits and Systems (APCCAS) ,ISBN no. 978-1-42447456-1, pp.772-775, Oct.2010.

[27] De-Shiuan Chiou, Yu-Ting Chen, Da-Cheng Juan, and Shih-Chieh, "Sleep Transistor Sizing for Leakage Power Minimization Considering Temporal Correlation", IEEE transactions on computer-aided design of integrated circuits and systems, vol. 29, no. 8,pp.1285-1290, August 2010 .

[28] Young-Kyun Cho, Young-Deuk Jeon, Jae-Won Nam, and Jong-Kee Kwon, "A 9-bit $80 \mathrm{MS} / \mathrm{s}$ Successive Approximation Register Analog-to-Digital Converter With a Capacitor Reduction Technique" IEEE Circuits And Systems, ISSN: 1549-7747 pp 502-506 Vol. 57, Issue:7, July 2010.

[29] R. Jacob Baker "CMOS: Mixed Signal Circuit Design", Second Edition, Wiley-IEEE Press, ISBN 978 047029026-2, 2009.

[30] A.B. Bhattacharyya "Compact MOSFET Models For VLSI Design" John Wiley \& Sons, ISBN: 978-0-47082342-2, 2009.

[31] P.Iyappan, P.Jamuna and S.Vijayasamundiswary, "Design of Analog to Digital Converter Using CMOS Logic", IEEE International Conference on Advances in Recent Technologies in Communication and Computing, ISBN no. 978-0-7695-3845-7, pp. 74-76, 2009.
[32] Rajashekar G, M S Bhat "Design of Resolution Adaptive TIQ Flash ADC using AMS $0.35 \mu \mathrm{m}$ Technology" IEE International Conference on Electronic Design (ICED), ISSN 978-1-4244-2315-6, pp.1-6, Dec-2008.

[33] O.Aytar, A.Tangel, G. Dundar, "A 9-Bit 1GS/S CMOS Folding ADC Implementation Using TIQ Based Flsh ADC Cores" MIXDES $15^{\text {th }}$ Int. Conference 2008, Print ISBN: 978-83-922632-7-2 Poznan, Poland, pp 159-164, Oct-2008.

[34] Erik Sall and Mark Vesterbacka, "Thermometer-toBinary Decoders for Flash Analog-to-Digital Converters", European Conference on Circuit Theory and Design - ECCTD,ISBN no.1-4244-1342-7, pp. 240243, July2007.

[35] Mingzhen Wang, Chien-In Henry Chen, and Shailesh Radhakrishnan,"Low-Power 4-b 2.5-GSPS Pipelined Flash Analog-to-Digital Converter in 130-nm CMOS", IEEE Transactions on Instrumentation and Measurement, Vol. 56, no. 3, pp. 1064- 1073, June 2007.

[36] Kaijian Shi David Howard , "Challenges in Sleep Transistor Design and Implementation in Low-Power Designs”,pp.113-116,July 2006.

[37] Ferragina, V.; Ghittori, N.; Maloberti, F., "Low-power 6bit flash ADC for high-speed data converters architectures", IEEE International Symposium on circuits and Systems (ISCAS), Print ISBN: 0-7803-93899, pp. 3930-3933, Sep- 2006.

[38] Jincheol Yoo,Kyusun Choi and Jahan Ghaznavi, "A A $0.07 \mu \mathrm{m}$ CMOS Flash Analog-to-Digital Converter for High Speed and Low Voltage Applications", GLSVLSI, pp.56-59,2003.

[39] Xuemei (Jane) Xi, Mohan Dunga, Jin He, Weidong Liu, Kanyu M. Cao, Xiaodong Jin, Jeff Jou, Mansun Chan, Ali M. Niknejad, Chenming Hu "BSIM4.3.0 MOSFET Model: User Manual", 2003.

[40] P. E. Allen and D. R. Holberg. "CMOS Analog Circuit Design" second edition oxford university press. ISBN 0195116445,2002

[41] D. Lee, J. Yoo, and K. Choi. "Design Method and Automation of Comparator Generation for Flash A/D Converters". IEEE International Symposium on Quality Electronic Design, pp.138-142, 2002.

[42] D. Lee, J. Yoo, K. Choi, and J. Ghaznavi. "Fat Tree Encoder Design for Ultra-High Speed Flash A/D Converters", IEEE Midwest Symposium on Circuits and Systems, 2002. 